CIENCIAMATRIA

Revista Interdisciplinaria de Humanidades, Educación, Ciencia y Tecnología

Año VI. Vol. VI. N 1 . Edición Especial. 2020

Hecho el depósito de ley: pp201602FA4721

ISSN-L: 2542-3029; ISSN: 2610-802X

Universidad Nacional Experimental Francisco de Miranda (UNEFM). Santa Ana de Coro. Venezuela

Aida Monserrate Macias-Alvia; Tibisay Rincón-Ríos; Bolívar Cevallos-Jácome; Letty Soraida Quimi-Cobos

DOI 10.35381/cm.v6i1.383

\title{
Capacidad de agencia de autocuidado de la salud en docentes de la Carrera de Enfermería
}

\section{Agency capacity of self-care of health in teachers of the Nursing Career}

\author{
Aida Monserrate Macías-Alvia \\ aidita.macias@hotmail.com \\ Universidad Estatal del Sur de Manabí, Jipijapa \\ Ecuador \\ https://orcid.org/0000-0001-5290-4317 \\ Tibisay Rincón-Ríos \\ tibyrin@hotmail.com \\ Universidad del Zulia, Zulia \\ Venezuela \\ https://orcid.org/0000-0003-4288-1822 \\ Bolívar Cevallos-Jácome \\ boly1404@gmail.com \\ Universidad Técnica de Manabí, Portoviejo \\ Ecuador \\ https://orcid.org/0000-0002-6334-1385 \\ Letty Soraida Quimi-Cobos \\ lettyquimic63@hotmail.com \\ Universidad Estatal del Sur de Manabí, Jipijapa \\ https://orcid.org/0000-0002-8577-5205
}

Recibido: 01 de agosto de 2020

Aprobado: 15 de octubre de 2020 


\title{
RESUMEN
}

Objetivo: Describir la capacidad de agencia de autocuidado de la salud en docentes de la carrera de Enfermería de la Universidad Estatal del Sur de Manabí. Metodología: De tipo cuantitativo descriptivo transversal y con un diseño no experimental, la población estuvo constituida por 14 docentes de la carrera de Enfermería de la Universidad Estatal del Sur de Manabí. Conclusión: La capacidad de agencia de autocuidado es deficiente en la mitad de los participantes, al lograr ubicarse entre el nivel medio y bajo de evaluación. Asimismo, el total de los docentes participan en reuniones o festividades sociales, comparten actividades de recreación con su familia y nunca sustituyen la comunicación directa con su familia por el uso del chat, teléfono o celular.

Descriptores: Autocuidado; docentes; enfermería; Atención Primaria de Salud. (Fuente DeCS, 2020).

\begin{abstract}
Objective: to describe the capacity of health self-care agency in teachers of the Nursing career of the Southern State University of Manabí. Methodology: Of a transverse descriptive quantitative type and with a non-experimental design, the population consisted of 14 teachers of the nursing career of the Southern State University of Manabí. Conclusion: Self-care agency capacity is poor in half of participants, as they are between medium and low level of assessment. In addition, the total number of teachers participate in meetings or social festivities, share recreation activities with their family and never replace direct communication with their family with the use of chat, phone or cell phone.
\end{abstract}

Descriptors: Self-care; teacher; nursing; primary Health Care. (Source DeCS, 2020). 
Universidad Nacional Experimental Francisco de Miranda (UNEFM). Santa Ana de Coro. Venezuela

Aida Monserrate Macias-Alvia; Tibisay Rincón-Ríos; Bolívar Cevallos-Jácome; Letty Soraida Quimi-Cobos

\section{INTRODUCCIÓN}

La enfermería es una profesión del sistema de salud focalizada en el cuidado de los individuos, las familias y las comunidades para que puedan alcanzar, mantener o recobrar una salud óptima y una calidad de vida adecuada ${ }^{1}$. Cabe destacar, que la existencia de un déficit de autocuidado se da cuando la demanda de acción es mayor que la capacidad de las personas para actuar, incapacitándola para el autocuidado continuo ${ }^{2}$,avalando la necesidad de los cuidados de Enfermería.

Algunas investigaciones apuntan a que un déficit en las capacidades de autocuidado aumentan el riesgo de enfermedades crónicas no transmisibles en los estudiantes universitarios y el número de años de vida sana perdidos alcanza hasta 6,9 años en algunas regiones de Latinoamérica ${ }^{3}$. De allí, la necesidad de contar con docentes que promuevan las capacidades de autocuidado a la vez que desarrollen competencias profesionales de los estudiantes durante su formación. El cuidado como repercusión en la práctica ${ }^{5}$, requiere ser analizado desde el punto de vista epistemológico del conocimiento enfermero y de las distintas expresiones y significados de la persona que lo recibe como ser humano integral, hasta los significados que simbolizan para los enfermeros (as) que lo brindan. En el contexto de los modelos conceptuales de las teorizantes de enfermería es necesario introducir otras teorías que permitan la producción del conocimiento, como punto de partida para la construcción de la ciencia ${ }^{5}$. Es por ello que el proceso de autocuidado empieza cuando se toma conciencia de cuidar de uno mismo, con el fin de mejorar el estado de salud convirtiéndose esto en una tarea constante; es así como aquellos individuos que tenga la capacidad para satisfacer sus propias necesidades tendrán mayor agencia de autocuidado ${ }^{6}$.

De allí que, el docente de enfermería en su rol de educar, no implica sólo socializar contenidos, sino introducirse en el significado de la cultura en la cual está inmerso el educando y contextualizarse en sus propias estructuras especiales, en ese sentido, la filosofía de educar y la pedagogía del cuidado son una misma ${ }^{7}$. Con base a la 
Universidad Nacional Experimental Francisco de Miranda (UNEFM). Santa Ana de Coro. Venezuela

Aida Monserrate Macias-Alvia; Tibisay Rincón-Ríos; Bolívar Cevallos-Jácome; Letty Soraida Quimi-Cobos problemática expuesta, se propone en este artículo describir la capacidad de agencia de autocuidado de la salud en docentes de la carrera de Enfermería de la Universidad Estatal del Sur de Manabí.

\section{DESARROLLO}

\section{Agenda del Autocuidado}

Es una característica humana compleja y adquirida que le da poder a un individuo para comprometerse en las operaciones esenciales de autocuidado ${ }^{8}$. El ejercicio por parte de un individuo, del poder llamado agencia de autocuidado, resulta en un sistema de acciones direccionadas hacia condiciones de la realidad del entorno, a fin de regularlas. La agencia de autocuidado puede conceptualizarse como un repertorio de acción de un individuo o ser caracterizada en términos de habilidades y limitaciones de un individuo para comprometerse en el autocuidado ${ }^{9}$.

Esta estructura conceptual de acción intencionada comprende operaciones estimativas, transicionales de reflexión (incluyen el juicio crítico y la toma de decisiones) y producción de operaciones buscando satisfacer los requisitos de autocuidado ${ }^{9}$. Las operaciones estimativas y transicionales comprenden la apropiación de conocimientos, actitudes y practicas efectivas y convenientes para disponer de las habilidades necesarias dando un curso de acción a la conducta de autocuidado más apropiada ${ }^{9}$.

Por otro lado, las habilidades que impulsan la acción de la agencia de autocuidado según Orem, citado por Landeros ${ }^{10}$, están definidas por los siguientes componentes: 1. Habilidad para mantener la atención con respecto a uno mismo como agente de autocuidado y de los factores externos. 2. Habilidad para controlar el uso de energía. 3. Habilidad para controlar la posición corporal. 4. Habilidad para razonar dentro de un marco de autocuidado. 5. Motivación al autocuidado. 6. Habilidad para tomar decisiones sobre el cuidado de uno mismo. 7. Habilidad para adquirir conocimiento técnico sobre autocuidado. 8. Repertorio de habilidades diversas para llevar a cabo operaciones de 
Aida Monserrate Macias-Alvia; Tibisay Rincón-Ríos; Bolívar Cevallos-Jácome; Letty Soraida Quimi-Cobos

autocuidado. 9. Habilidad para ordenar acciones de autocuidado. 10. Habilidad para integrar el autocuidado en la vida individual, familiar y comunitaria ${ }^{10}$.

A tal efecto, la agencia de autocuidado se construye y se desarrolla, a través de los ciclos vitales de los individuos asociados al deseo humano de conocer, aprender y llegar a un desarrollo personal cada vez más elevado; apreciaciones que el científico debe determina durante su medición ${ }^{9}$.

De allí que, para entender el concepto de agencia de autocuidado, se deben identificar las capacidades humanas para el desempeño de ciertas operaciones de autocuidado y su resultado, por un lado las operaciones de investigación buscan conocimientos prácticos y técnicos, con el fin de entender exactamente que es el cuidado de sí mismo y cómo hacerlo; las operaciones transicionales se relacionan con la capacidad de considerar, conceptuar y resolver los asuntos de autocuidado, sus experiencias y requisitos para satisfacerlo; por último las operaciones productivas se relacionan con las medidas de autocuidado, como realizarlas y controlarlas para tener claro los efectos y resultados de estas ${ }^{6}$.

Por otra parte, el concepto básico desarrollado por Orem ${ }^{11} \mathrm{del}$ autocuidado se define como el conjunto de acciones intencionadas que realiza la persona para controlar los factores internos o externos, que pueden comprometer su vida y desarrollo posterior. El autocuidado por tanto, es una conducta que realiza o debería realizar la persona para sí misma, consiste en la práctica de las actividades que las personas maduras, o que están madurando, inician y llevan a cabo en determinados períodos de tiempo, por su propia parte y con el interés de mantener un funcionamiento vivo y sano, continuar además, con el desarrollo personal y el bienestar mediante la satisfacción de requisitos para las regulaciones funcional y del desarrollo ${ }^{12}$. También ${ }^{13}$, plantean que el eje fundamental de la enfermería es identificar el déficit entre la capacidad potencial de autocuidado y las demandas de autocuidado de los pacientes. La meta es eliminar este, de tal forma que se cubran los requerimientos/necesidades universales del desarrollo y se limiten las 
Aida Monserrate Macias-Alvia; Tibisay Rincón-Ríos; Bolívar Cevallos-Jácome; Letty Soraida Quimi-Cobos desviaciones en la salud.

Según lo antes expuesto se asume el autocuidado como la responsabilidad que tiene cada individuo para el fomento, conservación y cuidado de su propia salud. Por lo que se hace necesaria la individualización de los cuidados y la implicación de los usuarios en el propio plan de cuidados, y otorga protagonismo al sistema de preferencias del sujeto. Por otro lado supone trabajar con aspectos relacionados con la motivación y cambio de comportamiento, y hacer de la educación para la salud la herramienta principal de trabajo 14.

\section{Atención primaria en salud}

Refiere a la asistencia sanitaria esencial basada en métodos y tecnologías prácticos, científicamente fundados y socialmente aceptables, puesta al alcance de todos los individuos y familias de la comunidad mediante su plena participación y a un costo que la comunidad y el país puedan soportar, en todas y cada una de las etapas de su desarrollo con un espíritu de autorresponsabilidad y autodeterminación, para la Organización Mundial de la Salud (OMS) ${ }^{15}$ es la siguiente. La atención primaria forma parte integrante tanto del sistema nacional de salud, del que constituye la función central y el núcleo principal, como del desarrollo social y económico global de la comunidad ${ }^{16}$, la misma debe ofrecer servicios según la necesidad, y dar respuesta en su nivel a la mayoría de los problemas. En tal sentido, se debe contar con una atención primaria polivalente y resolutiva, competente para dar respuesta a pacientes complejos y para considerar la salud en conjunto, y que coopere con otros servicios, socio-sanitarios y extra-sanitarios 10.

\section{La enseñanza del autocuidado}

Se indica ${ }^{17}$ que el autocuidado se desarrolla de manera aprendida, en todas las etapas evolutivas del ser humano, fomentando capacidades para realizar acciones 
Aida Monserrate Macias-Alvia; Tibisay Rincón-Ríos; Bolívar Cevallos-Jácome; Letty Soraida Quimi-Cobos intencionadas que lo involucran, tomando decisiones sobre qué hacer y qué no. A medida que pasa el tiempo; los individuos deben tener un proceso de aprendizaje y aplicarlo para realizar acciones orientadas al cuidado interno y externo; generalmente la edad, el estado de desarrollo y la salud determinan el alcance de las acciones dirigidas al autocuidado, así como los valores y los objetivos que se tienen y que permiten dirigir hacia el cuidado de la salud y la prevención de la enfermedad ${ }^{18}$.

En este sentido, la práctica del autocuidado en los docentes es un factor clave para los establecimientos educacionales, ya que impacta directamente en la calidad de sus procesos educativos, pero no hablamos sólo de la salud física, la docencia también implica un alto desgaste emocional ${ }^{16}$. Es imprescindible que los profesores se hagan cargo de su realidad laboral e incorporen a su rutina el autocuidado, lo que finalmente impactará positivamente en su calidad de vida y la garantía de un buen proceso de enseñanza del mismo, entendiendo que se encuentran permanentemente profesores están arriesgados a un permanente estrés, de allí la importancia de tener presente un buen manejo del autocuidado ${ }^{14}$.

\section{Teorías de Orem que sustentan el autocuidado}

Exponen, que la teoría de Orem ${ }^{18}$ está sustentada en base a tres conceptos:

El autocuidado: en ella se explica y describe el autocuidado. Lo define como una actividad aprendida por la persona a lo largo de su vida y orientada hacia un objetivo. Es, por tanto, una conducta ante la vida dirigida hacia uno mismo en beneficio de la salud y el bienestar ${ }^{18}$. Por tanto, la persona que es autosuficiente es responsable de sus propios cuidados y de los de las personas que tiene a su cargo y tendrá capacidades para dar respuesta a toda una serie de necesidades para la vida. Ello debe suponer la capacidad de llevar una vida sana, comportarse de forma que se trate de alcanzar el bienestar y la salud ${ }^{8}$. 
Aida Monserrate Macias-Alvia; Tibisay Rincón-Ríos; Bolívar Cevallos-Jácome; Letty Soraida Quimi-Cobos

Déficit de autocuidado: se da cuando la demanda de acción es mayor que la capacidad de las personas para actuar, incapacitándola para el autocuidado continúo. La existencia de un déficit de autocuidado es la condición que avala la necesidad de los cuidados de Enfermería ${ }^{8}$.

Los sistemas de Enfermería: Describen y explican las relaciones que son necesarias para garantizar al sistema como un complejo método de acciones realizadas por las enfermeras/os en atención a las demandas de autocuidado terapéutico del paciente ${ }^{18}$.

\section{MATERIALES Y MÉTODO}

Se trató de un enfoque cuantitativo descriptivo transversal ${ }^{19}$ y con un diseño no experimental. La población estuvo constituida por 14 docentes de la carrera de Enfermería de la Universidad Estatal del Sur de Manabí. La investigación mantuvo los parámetros éticos para su desarrollo ${ }^{20}$, la incorporación de los participantes se hizo después de la explicación precisa del propósito del estudio, y a quienes se les pidió su autorización para la aplicación del instrumento, los datos proporcionados se consideraron como anónimos y confidenciales. Se utilizó la escala ASA ("Apreciación de la agencia de autocuidado", por sus siglas en inglés), desarrollada en 1993 por Isenberg y Evers para medir el concepto central de la Teoría de Enfermería de Déficit de Autocuidado de Orem 18. La base de datos y el análisis estadístico de la información se realizaron en el programa SPSS versión 23.0 para calcular la estadística frecuencial y descriptiva. LoS resultados se presentaron en tablas con sus respectivos gráficos y análisis cuantitativo.

\section{RESULTADOS}

Con el fin de dar cumplimiento al objetivo planteado en el presente artículo, el cual es describir la capacidad de agencia de autocuidado de la salud en docentes de la carrera de Enfermería de la Universidad Estatal del Sur de Manabí, se presentan los resultados 
CIENCIAMATRIA

Revista Interdisciplinaria de Humanidades, Educación, Ciencia y Tecnología

Año VI. Vol. VI. N¹. Edición Especial. 2020

Hecho el depósito de ley: pp201602FA4721

ISSN-L: 2542-3029; ISSN: 2610-802X

Universidad Nacional Experimental Francisco de Miranda (UNEFM). Santa Ana de Coro. Venezuela

Aida Monserrate Macias-Alvia; Tibisay Rincón-Ríos; Bolívar Cevallos-Jácome; Letty Soraida Quimi-Cobos

del instrumento aplicado a los docentes de la carrera de Enfermería de la Universidad Estatal del Sur de Manabí, en tablas con sus respectivos gráficos e interpretación.

Tabla 1.

Análisis frecuencial y porcentual de la categoría interacción social en el autocuidado de la salud en docentes de la carrera de Enfermería de la Universidad Estatal del Sur de Manabí.

\begin{tabular}{|l|l|r|r|r|c|}
\hline Categoría/ Análisis & Nunca & $\begin{array}{l}\text { Raras } \\
\text { Veces }\end{array}$ & $\begin{array}{l}\text { Algunas } \\
\text { Veces }\end{array}$ & A Menudo & Siempre \\
\cline { 2 - 6 } & $\mathrm{Fr} / \%$ & $\mathrm{Fr} / \%$ & $\mathrm{Fr} / \%$ & $\mathrm{Fr} / \%$ & $\mathrm{Fr} / \%$ \\
\hline $\begin{array}{l}\text { ¿Suele mantener una } \\
\text { comunicación interpersonal } \\
\text { satisfactoria? }\end{array}$ & $1 / 7 \%$ & $1 / 7 \%$ & $1 / 7 \%$ & $1 / 7 \%$ & $10 / 72 \%$ \\
\hline $\begin{array}{l}\text { ¿Participa en las reuniones o } \\
\text { festividades sociales? }\end{array}$ & $0 / 0 \%$ & $0 / 0 \%$ & $0 / 0 \%$ & $0 / 0 \%$ & $14 / 100 \%$ \\
\hline $\begin{array}{l}\text { ¿Comparte actividades de } \\
\text { recreación con su familia? }\end{array}$ & $0 / 0 \%$ & $0 / 0 \%$ & $0 / 0 \%$ & $0 / 0 \%$ & $14 / 100 \%$ \\
\hline $\begin{array}{l}\text { ¿Prefieres hacer uso del chat, } \\
\text { teléfono o celular antes de } \\
\text { favorecer la comunicación directa } \\
\text { con su familia? }\end{array}$ & $14 / 100 \%$ & $0 / 0 \%$ & $0 / 0 \%$ & $0 / 0 \%$ & \\
\hline $\begin{array}{l}\text { ¿Tiene momentos de reflexión y } \\
\text { meditación en su vida diaria? }\end{array}$ & $0 / 0 \%$ & $0 / 0 \%$ & $1 / 7 \%$ & $4 / 28 \%$ & $9 / 65 \%$ \\
\hline
\end{tabular}


CIENCIAMATRIA

Revista Interdisciplinaria de Humanidades, Educación, Ciencia y Tecnología

Año VI. Vol. VI. N${ }^{\circ}$. Edición Especial. 2020

Hecho el depósito de ley: pp201602FA4721

ISSN-L: 2542-3029; ISSN: 2610-802X

Universidad Nacional Experimental Francisco de Miranda (UNEFM). Santa Ana de Coro. Venezuela

Aida Monserrate Macias-Alvia; Tibisay Rincón-Ríos; Bolívar Cevallos-Jácome; Letty Soraida Quimi-Cobos

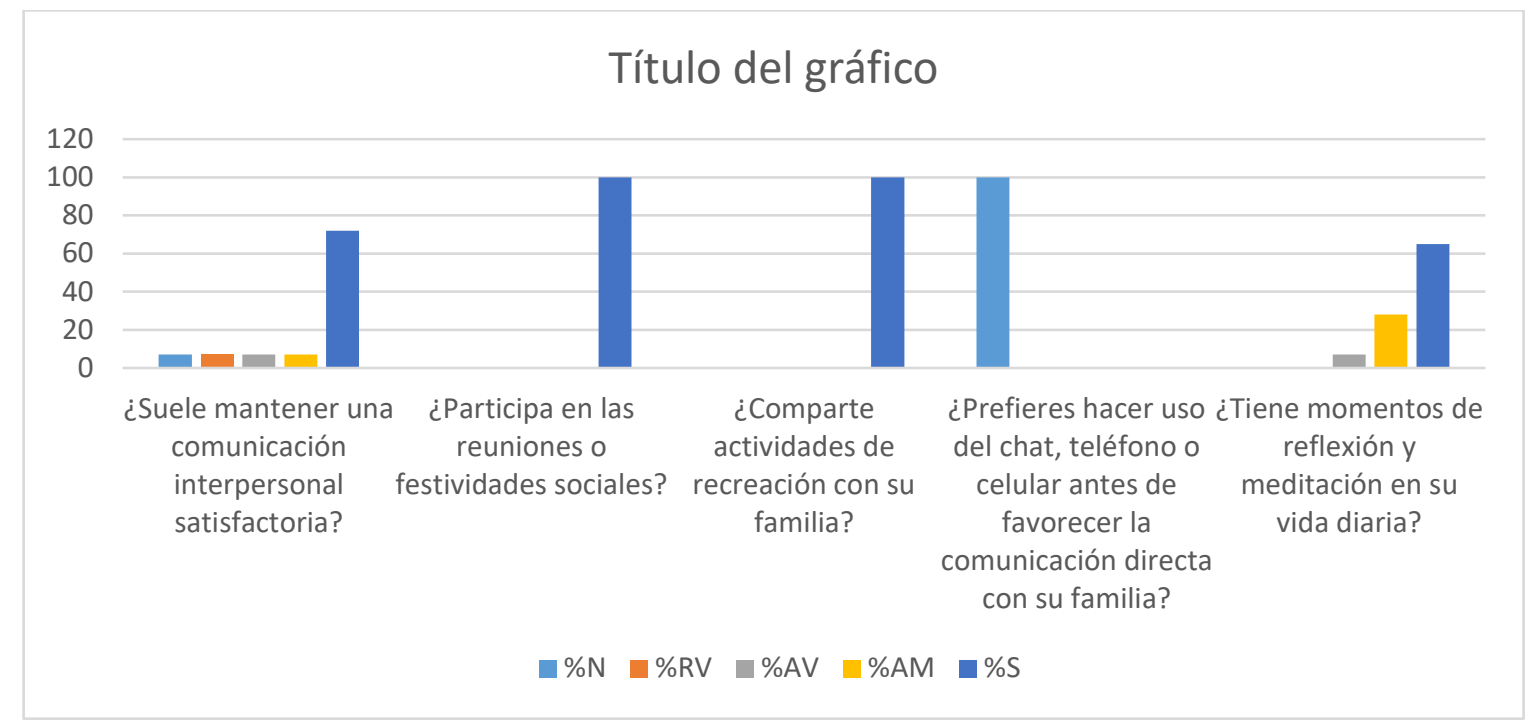

\section{Grafico 1.}

Análisis frecuencial y porcentual de la categoría interacción social en el autocuidado de la salud en docentes de la carrera de Enfermería de la Universidad Estatal del Sur de Manabí.

En la tabla y grafico No.1, se presenta el análisis frecuencial descriptivo de la categoría interacción social de la capacidad de agencia de autocuidado de la salud en docentes de la carrera de Enfermería de la Universidad Estatal del Sur de Manabí. En la misma se indica que el $100 \%$ del total de los docentes investigados coincidieron en opinar que siempre participan en las reuniones o festividades sociales, comparte actividades de recreación con su familia y nunca sustituirían favorecer la comunicación directa con su familia por el uso del chat, teléfono o celular. Seguido del $72 \%$ y $65 \%$ que indicaron la alternativa siempre hay interacción social al mantener una comunicación interpersonal satisfactoria y tener momentos de reflexión y meditación en la vida diaria, respectivamente.

\section{Tabla 2.}




\section{CIENCIAMATRIA}

Revista Interdisciplinaria de Humanidades, Educación, Ciencia y Tecnología

Año VI. Vol. VI. N${ }^{\circ}$. Edición Especial. 2020

Hecho el depósito de ley: pp201602FA4721

ISSN-L: 2542-3029; ISSN: 2610-802X

Universidad Nacional Experimental Francisco de Miranda (UNEFM). Santa Ana de Coro. Venezuela

Aida Monserrate Macias-Alvia; Tibisay Rincón-Ríos; Bolívar Cevallos-Jácome; Letty Soraida Quimi-Cobos

Análisis frecuencial y porcentual de la categoría prevención de riesgo en el autocuidado de la salud en docentes de la carrera de Enfermería de la Universidad Estatal del Sur de Manabí.

\begin{tabular}{|l|l|l|l|l|c|}
\hline Categoría/ Análisis & Nunca & $\begin{array}{l}\text { Raras } \\
\text { Veces }\end{array}$ & $\begin{array}{l}\text { Algunas } \\
\text { Veces }\end{array}$ & $\begin{array}{l}\text { A } \\
\text { Menudo }\end{array}$ & Siempre \\
\cline { 2 - 6 } & $\mathrm{Fr} / \%$ & $\mathrm{Fr} / \%$ & $\mathrm{Fr} / \%$ & $\mathrm{Fr} / \%$ & $\mathrm{Fr} / \%$ \\
\hline $\begin{array}{l}\text { ¿Se hace un autoexamen de } \\
\text { mamas, por lo menos una vez } \\
\text { al mes? }\end{array}$ & $1 / 7 \%$ & $2 / 14 \%$ & $3 / 21 \%$ & $4 / 29 \%$ & $4 / 29 \%$ \\
\hline $\begin{array}{l}\text { ¿Se realiza una mamografía y un } \\
\text { papanicolau cada año? }\end{array}$ & $8 / 56 \%$ & $1 / 7 \%$ & $1 / 7 \%$ & $1 / 7 \%$ & $3 / 21 \%$ \\
\hline $\begin{array}{l}\text { ¿En caso de ser varón visita al } \\
\text { urólogo con frecuencia? }\end{array}$ & $0 / 0 \%$ & $0 / 0 \%$ & $1 / 33.3 \%$ & $1 / 33.3 \%$ & $1 / 33,3 \%$ \\
\hline $\begin{array}{l}\text { ¿Se realiza exámenes médicos de } \\
\text { forma preventiva cada año? }\end{array}$ & $1 / 7 \%$ & $3 / 21 \%$ & $3 / 21 \%$ & $3 / 21 \%$ & $4 / 29 \%$ \\
\hline $\begin{array}{l}\text { ¿Consume con frecuencia } \\
\text { medicinas en general, sin recetas } \\
\text { médica? }\end{array}$ & $9 / 63 \%$ & $1 / 7 \%$ & $1 / 7 \%$ & $2 / 14 \%$ & $1 / 7 \%$ \\
\hline $\begin{array}{l}\text { ¿Visita al médico con frecuencia o } \\
\text { solo cuando está enfermo? }\end{array}$ & $8 / 56 \%$ & $1 / 7 \%$ & $1 / 7 \%$ & $2 / 14 \%$ & $2 / 14 \%$ \\
\hline Fuente: Elaboración propia $(2020)$ & & & & \\
\hline
\end{tabular}

Fuente: Elaboración propia (2020).

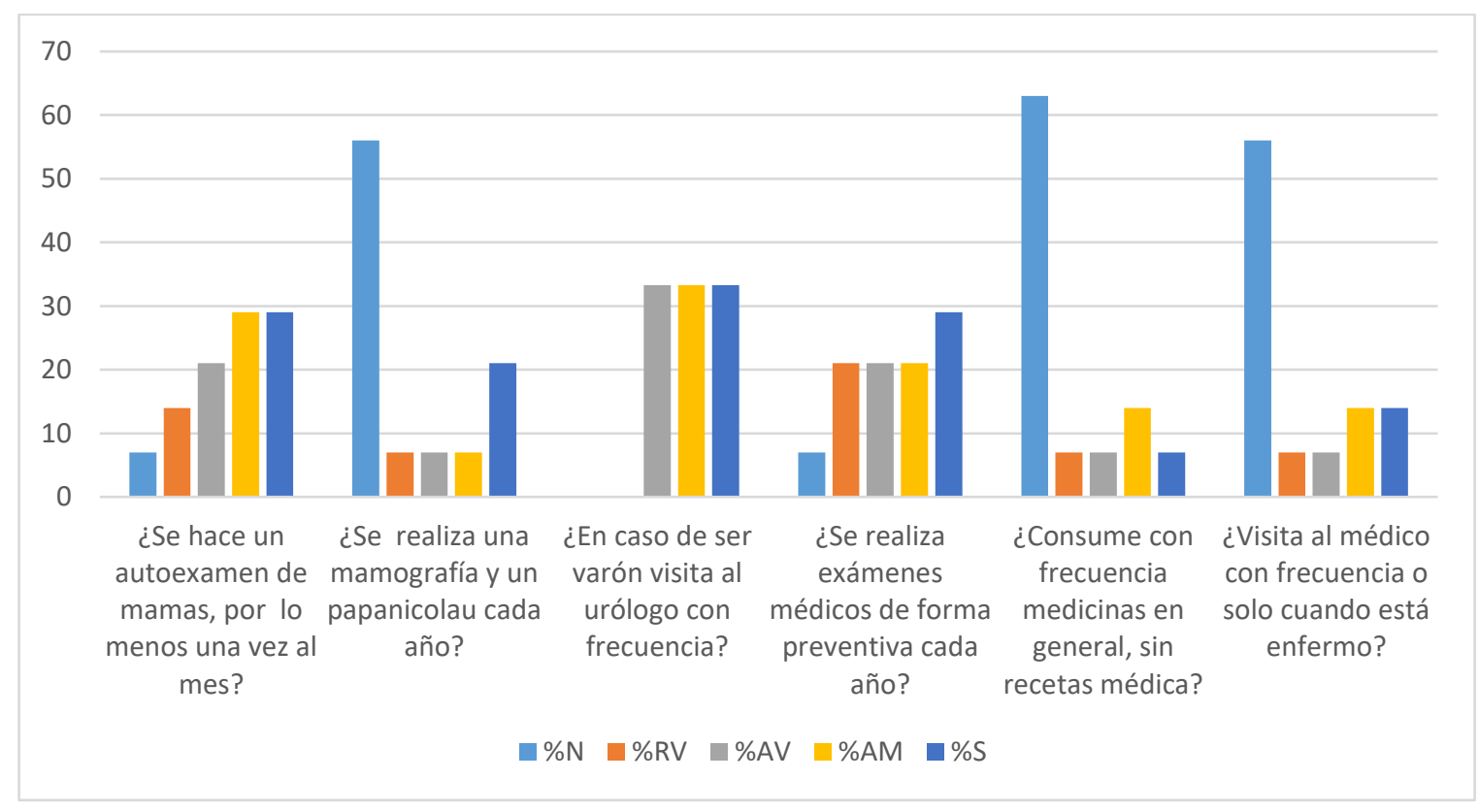


Aida Monserrate Macias-Alvia; Tibisay Rincón-Ríos; Bolívar Cevallos-Jácome; Letty Soraida Quimi-Cobos

\section{Grafico 2.}

Análisis frecuencial y porcentual de la categoría prevención de riesgo en el autocuidado de la salud en docentes de la carrera de Enfermería de la Universidad Estatal del Sur de Manabí

En la tabla y grafico No.2, se presenta el análisis frecuencial descriptivo de la categoría prevención del riesgo de la capacidad de agencia de autocuidado de la salud en docentes de la carrera de Enfermería de la Universidad Estatal del Sur de Manabí. En la misma el $63 \%$ de los docentes indicaron que no consumen con frecuencia medicinas en general, sin recetas médica y el $56 \%$ por igual nunca se han realizan una mamografía y un papanicolau durante un año ni visitan al médico con frecuencia. Seguido del $28 \%$ que siempre o a menudo se hacen un autoexamen de mamas, por lo menos una vez al mes y se realizan exámenes médicos de forma preventiva cada año. Entre los menores porcentajes se observa con el $21 \%$ se realiza una mamografía y un papanicolau cada año, por otra parte el 33,3\% de los varones investigados siempre, a menudo o algunas veces visitan al urólogo con frecuencia.

\section{Tabla 3.}

Análisis frecuencial y porcentual de la categoría actividad y reposo en el autocuidado de la salud en docentes de la carrera de Enfermería de la Universidad Estatal del Sur de Manabí.

\begin{tabular}{|l|l|l|l|l|c|}
\hline Categoría/ Análisis & Nunca & $\begin{array}{l}\text { Raras } \\
\text { Veces }\end{array}$ & $\begin{array}{l}\text { Algunas } \\
\text { Veces }\end{array}$ & A Menudo & Siempre \\
\cline { 2 - 6 } & $\mathrm{Fr} / \%$ & $\mathrm{Fr} / \%$ & $\mathrm{Fr} / \%$ & $\mathrm{Fr} / \%$ & $\mathrm{Fr} / \%$ \\
\hline $\begin{array}{l}\text { ¿Siente que duerme bien y se } \\
\text { levanta descansada? }\end{array}$ & $5 / 37 \%$ & $2 / 14 \%$ & $2 / 14 \%$ & $2 / 14 \%$ & $3 / 21 \%$ \\
\hline $\begin{array}{l}\text { ¿Le cuesta trabajo conciliar el } \\
\text { sueño? }\end{array}$ & $5 / 37 \%$ & $2 / 14 \%$ & $2 / 14 \%$ & $2 / 14 \%$ & $3 / 21 \%$ \\
\hline $\begin{array}{l}\text { ¿Realiza siestas diarias, en } \\
\text { promedio de 20 a 30 minutos? }\end{array}$ & & & & & \\
\hline
\end{tabular}

Fuente: Elaboración propia (2020). 
CIENCIAMATRIA

Revista Interdisciplinaria de Humanidades, Educación, Ciencia y Tecnología

Año VI. Vol. VI. N${ }^{\circ}$. Edición Especial. 2020

Hecho el depósito de ley: pp201602FA4721

ISSN-L: 2542-3029; ISSN: 2610-802X

Universidad Nacional Experimental Francisco de Miranda (UNEFM). Santa Ana de Coro. Venezuela

Aida Monserrate Macias-Alvia; Tibisay Rincón-Ríos; Bolívar Cevallos-Jácome; Letty Soraida Quimi-Cobos

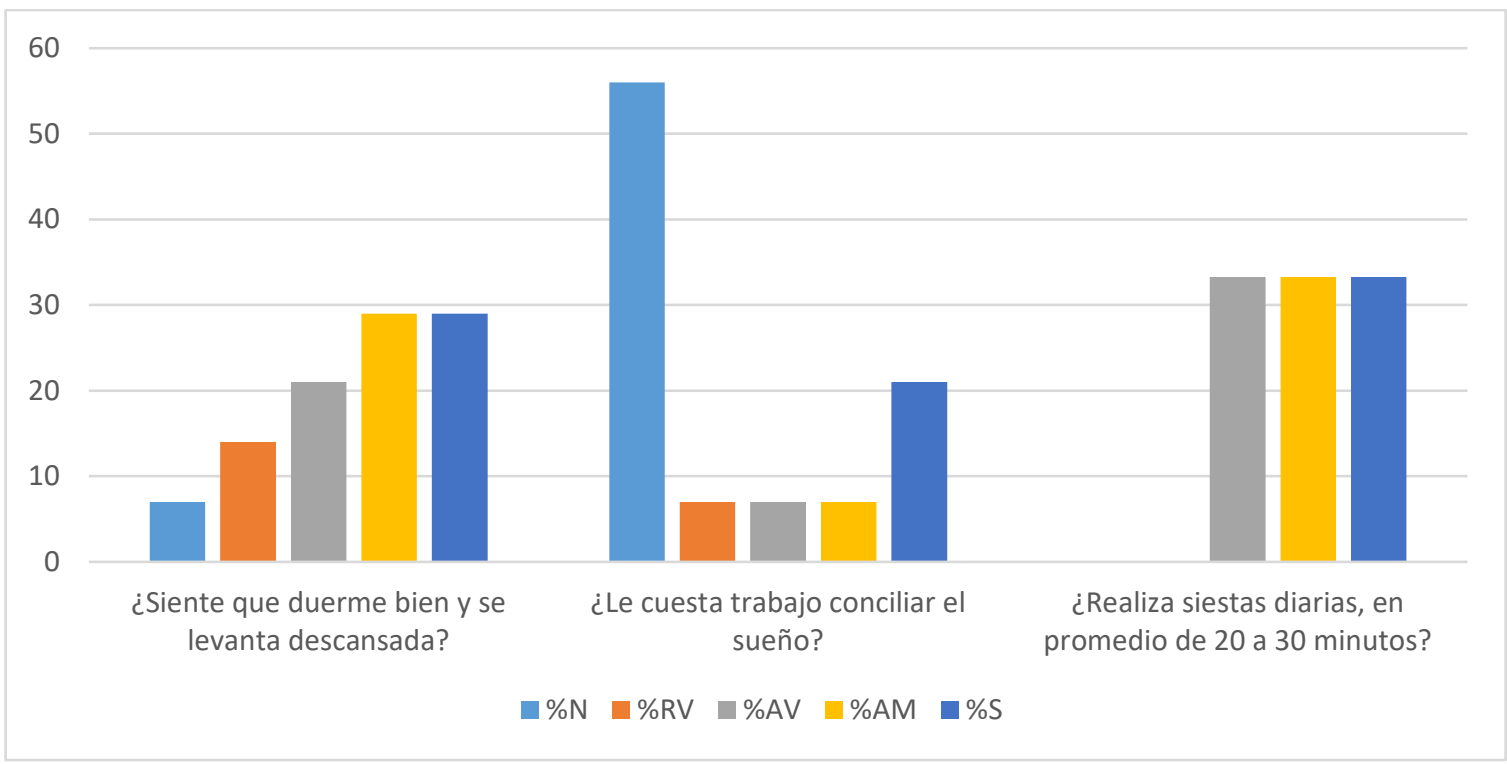

\section{Grafico 3.}

Análisis frecuencial y porcentual de la categoría actividad y reposo en el autocuidado de la salud en docentes de la carrera de Enfermería de la Universidad Estatal del Sur de Manabí.

En la tabla y grafico No.3, se presenta el análisis frecuencial descriptivo de la categoría actividad y reposo de la capacidad de agencia de autocuidado de la salud en docentes de la carrera de Enfermería de la Universidad Estatal del Sur de Manabí. En la misma se destaca que el $37 \%$ de los docentes investigados señalaron que nunca sienten que duerme bien y se levantan descansados o les cuesta trabajo conciliar el sueño y realizan siestas diarias, en promedio de 20 a 30 minutos. Seguido del $21 \%$ que señalaron la alternativa siempre lo cumplen y con menor porcentaje de solo el $14 \%$ indicaron las alternativas a menudo.

\section{Tabla 4.}




\section{CIENCIAMATRIA}

Revista Interdisciplinaria de Humanidades, Educación, Ciencia y Tecnología

Año VI. Vol. VI. N${ }^{\circ}$. Edición Especial. 2020

Hecho el depósito de ley: pp201602FA4721

ISSN-L: 2542-3029; ISSN: 2610-802X

Universidad Nacional Experimental Francisco de Miranda (UNEFM). Santa Ana de Coro. Venezuela

Aida Monserrate Macias-Alvia; Tibisay Rincón-Ríos; Bolívar Cevallos-Jácome; Letty Soraida Quimi-Cobos

Análisis frecuencial y porcentual de la categoría alimentos en el autocuidado de la salud en docentes de la carrera de Enfermería de la Universidad Estatal del Sur de Manabí.

\begin{tabular}{|l|l|c|c|c|c|}
\hline \multirow{2}{*}{ Categoría/ Análisis } & Nunca & $\begin{array}{l}\text { Raras } \\
\text { Veces }\end{array}$ & $\begin{array}{l}\text { Algunas } \\
\text { Veces }\end{array}$ & A Menudo & Siempre \\
\cline { 2 - 6 } & $\mathrm{Fr} / \%$ & $\mathrm{Fr} / \%$ & $\mathrm{Fr} / \%$ & $\mathrm{Fr} / \%$ & $\mathrm{Fr} / \%$ \\
\hline $\begin{array}{l}\text { ¿Mantiene un horario regular en las } \\
\text { comidas principales (D-A-C)? }\end{array}$ & $5 / 36 \%$ & $1 / 7 \%$ & $1 / 7 \%$ & $2714 \%$ & $5 / 36 \%$ \\
\hline $\begin{array}{l}\text { ¿Desayuna antes de iniciar su } \\
\text { actividad diaria? }\end{array}$ & $8 / 58 \%$ & $1 / 7 \%$ & $1 / 7 \%$ & $1 / 7 \%$ & $3 / 21 \%$ \\
\hline $\begin{array}{l}\text { Su alimentación ¿incluye pescados, } \\
\text { raciones de verduras, cereales, } \\
\text { menestras y productos lácteos mínimo } \\
\text { tres veces por semana? }\end{array}$ & & & & & \\
\hline $\begin{array}{l}\text { ¿Consume carnes rojas más de tres } \\
\text { veces a la semana? }\end{array}$ & $7 / 51 \%$ & $2 / 14 \%$ & $2 / 14 \%$ & $2 / 14 \%$ & $1 / 7 \%$ \\
\hline $\begin{array}{l}\text { ¿Consume con frecuencia pizzas, } \\
\text { hamburguesas, salchipapas y } \\
\text { productos que contengan azúcar } \\
\text { (golosinas) etc? }\end{array}$ & & & & & \\
\hline $\begin{array}{l}\text { ¿Consume mínimo dos tipos de frutas } \\
\text { al día y mantiene su requerimiento } \\
\text { nutricional diario? 1900 Kcal - 2200 } \\
\text { Kcal? }\end{array}$ & $10 / 72 \%$ & $1 / 7 \%$ & $1 / 7 \%$ & $1 / 7 \%$ & $1 / 7 \%$ \\
\hline
\end{tabular}

\section{Fuente: Elaboración propia (2020)}

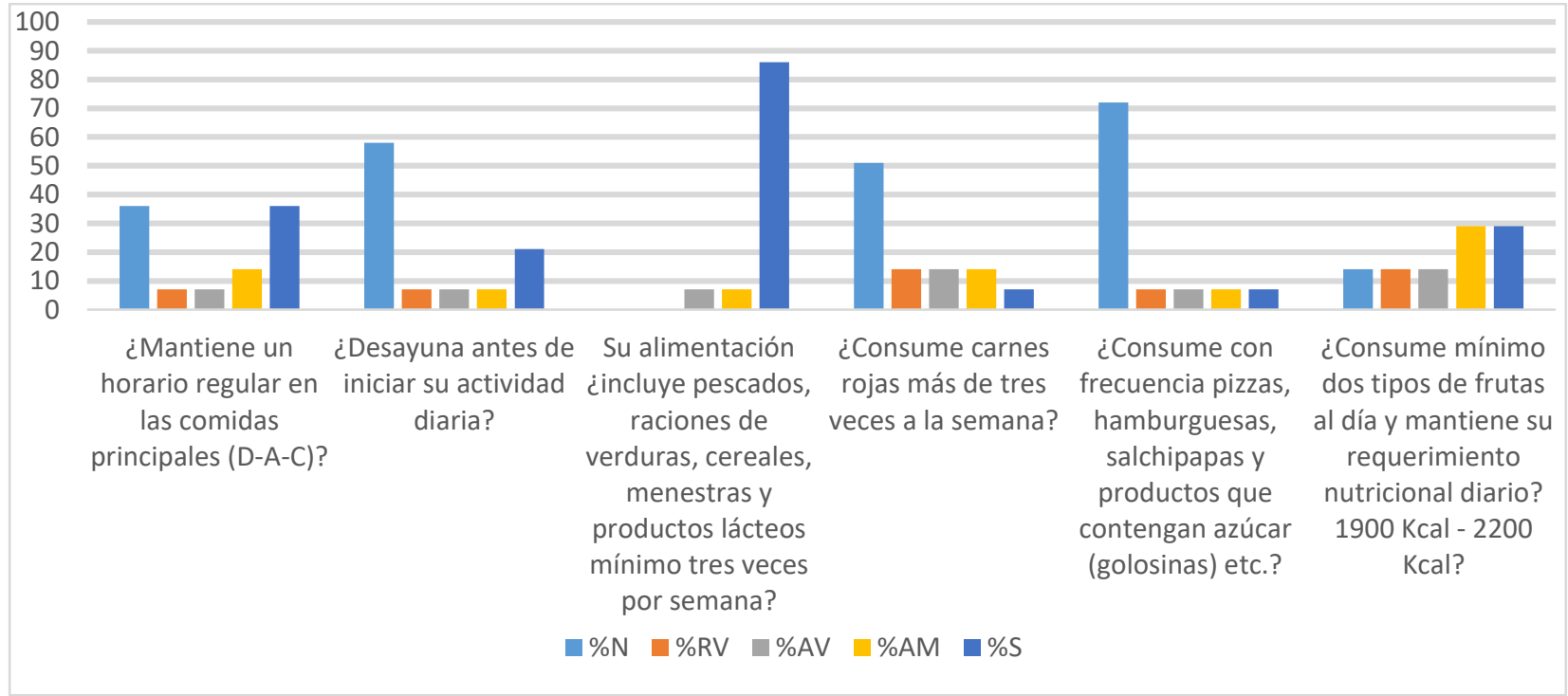




\section{CIENCIAMATRIA}

Revista Interdisciplinaria de Humanidades, Educación, Ciencia y Tecnología

Año VI. Vol. VI. N${ }^{\circ}$. Edición Especial. 2020

Hecho el depósito de ley: pp201602FA4721

ISSN-L: 2542-3029; ISSN: 2610-802X

Universidad Nacional Experimental Francisco de Miranda (UNEFM). Santa Ana de Coro. Venezuela

Aida Monserrate Macias-Alvia; Tibisay Rincón-Ríos; Bolívar Cevallos-Jácome; Letty Soraida Quimi-Cobos

\section{Grafico 4.}

Análisis frecuencial y porcentual de la categoría alimentos en el autocuidado de la salud en docentes de la carrera de Enfermería de la Universidad Estatal del Sur de Manabí.

En la tabla y grafico No.4, se presenta el análisis frecuencial descriptivo de la categoría alimentos para la capacidad de agencia de autocuidado de la salud en docentes de la carrera de Enfermería de la Universidad Estatal del Sur de Manabí. En la misma el 86\% indico que siempre su alimentación incluye pescados, raciones de verduras, cereales, menestras y productos lácteos mínimo tres veces por semana y el $72 \%$ de los docentes indicaron que no consumen con frecuencia pizzas, hamburguesas, salchipapas y productos que contengan azúcar (golosinas). Un 58\% indico que nunca desayunan antes de iniciar su actividad diaria y el $51 \%$ nunca consume carnes rojas más de tres veces a la semana. Los menores porcentajes se indicaron para las alternativas extremas de siempre y nunca con el $36 \%$ por igual mantienen un horario regular en las comidas principales de desayuno, almuerzo y cena y el $29 \%$ que opino en a menudo y siempre consumen mínimo dos tipos de frutas al día y mantiene su requerimiento nutricional diario entre 1900 Kcal - 2200 Kcal.

\begin{tabular}{|l|l|l|l|l|c|}
\hline Categoría/ Análisis & Nunca & $\begin{array}{l}\text { Raras } \\
\text { Veces }\end{array}$ & $\begin{array}{l}\text { Algunas } \\
\text { Veces }\end{array}$ & A Menudo & Siempre \\
\cline { 2 - 6 } & $\mathrm{Fr} / \%$ & $\mathrm{Fr} / \%$ & $\mathrm{Fr} / \%$ & $\mathrm{Fr} / \%$ & $\mathrm{Fr} / \%$ \\
\hline $\begin{array}{l}\text { ¿Hago ajustes en mis hábitos de vida } \\
\text { para } \\
\text { Mantenerme saludable? }\end{array}$ & $2 / 14 \%$ & $2 / 14 \%$ & $2 / 14 \%$ & $4 / 29 \%$ & $4 / 29 \%$ \\
\hline $\begin{array}{l}\text { ¿Tengo la capacidad de manejar las } \\
\text { situaciones para no perder el }\end{array}$ & & $0 / 0 \%$ & $0 / 0 \%$ & $6 / 42 \%$ & $8 / 58 \%$ \\
\hline
\end{tabular}


CIENCIAMATRIA

Revista Interdisciplinaria de Humanidades, Educación, Ciencia y Tecnología

Año VI. Vol. VI. N${ }^{\circ}$. Edición Especial. 2020

Hecho el depósito de ley: pp201602FA4721

ISSN-L: 2542-3029; ISSN: 2610-802X

Universidad Nacional Experimental Francisco de Miranda (UNEFM). Santa Ana de Coro. Venezuela

Aida Monserrate Macias-Alvia; Tibisay Rincón-Ríos; Bolívar Cevallos-Jácome; Letty Soraida Quimi-Cobos

\begin{tabular}{|l|c|c|c|c|c|}
\hline $\begin{array}{l}\text { control y mantenerme en } \\
\text { equilibrio? }\end{array}$ & $0 / 0 \%$ & & & \\
\hline $\begin{array}{l}\text { ¿Tomo medidas para asegurar que } \\
\text { mi familia y yo estemos seguros? }\end{array}$ & $0 / 0 \%$ & $0 / 0 \%$ & $0 / 0 \%$ & $0 / 0 \%$ & $14 / 100 \%$ \\
\hline $\begin{array}{l}\text { ¿Aun con problemas de salud ¿́soy } \\
\text { capaz de cuidarme? }\end{array}$ & $1 / 7 \%$ & $1 / 7 \%$ & $1 / 7 \%$ & $2 / 14 \%$ & $9 / 65 \%$ \\
\hline
\end{tabular}

Tabla 5.

Análisis frecuencial y porcentual de la categoría promoción del funcionamiento y desarrollo personal dentro del grupo social en el autocuidado de la salud en docentes de la carrera de Enfermería de la Universidad Estatal del Sur de Manabí.

Fuente: Elaboración propia (2020).

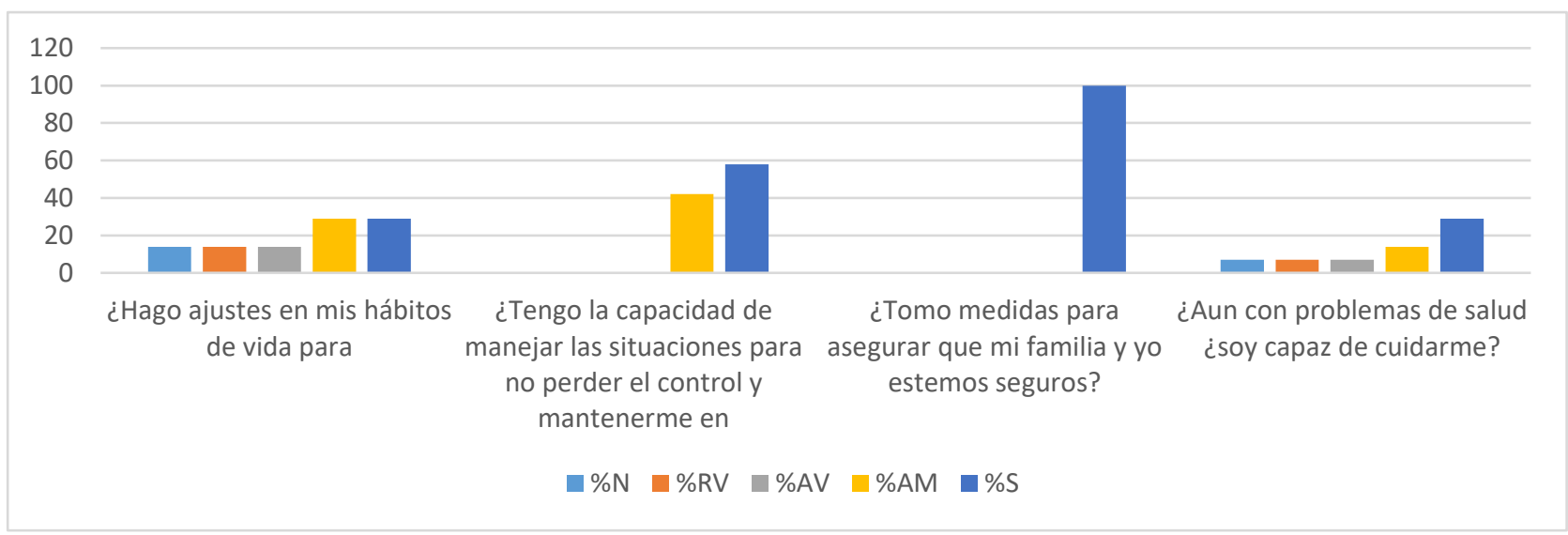

\section{Grafico 5.}

Análisis frecuencial y porcentual de la categoría promoción del funcionamiento y desarrollo personal dentro del grupo social en el autocuidado de la salud en docentes de la carrera de Enfermería de la Universidad Estatal del Sur de Manabí.

En la tabla y grafico No.5, se presenta el análisis frecuencial descriptivo de la categoría promoción del funcionamiento y desarrollo personal dentro del grupo social en el autocuidado de la salud en docentes de la carrera de Enfermería de la Universidad Estatal del Sur de Manabí. En la misma se destaca que el $100 \%$ de los docentes 
CIENCIAMATRIA

Revista Interdisciplinaria de Humanidades, Educación, Ciencia y Tecnología

Año VI. Vol. VI. N${ }^{\circ}$. Edición Especial. 2020

Hecho el depósito de ley: pp201602FA4721

ISSN-L: 2542-3029; ISSN: 2610-802X

Universidad Nacional Experimental Francisco de Miranda (UNEFM). Santa Ana de Coro. Venezuela

Aida Monserrate Macias-Alvia; Tibisay Rincón-Ríos; Bolívar Cevallos-Jácome; Letty Soraida Quimi-Cobos

investigados señalaron que siempre toman medidas para asegurar que su familia y su persona estén seguros. Seguido del $65 \%$ que señalaron que siempre aun con problemas de salud son capaces de cuidarse y el 58\% tienen la capacidad de manejar las situaciones para no perder el control y mantenerme en equilibrio y capaces de cuidarse. Con menor porcentaje de un $29 \%$ por igual siempre o a menudo tienen la capacidad de manejar las situaciones para no perder el control y mantenerme en equilibrio.

\section{Tabla 6.}

Distribución frecuencial y porcentual según agencia de autocuidado del total de los docentes de la carrera de Enfermería de la Universidad Estatal del Sur de Manabí.

\begin{tabular}{|c|c|c|}
\hline $\begin{array}{c}\text { Capacidad agencia } \\
\text { de autocuidado }\end{array}$ & FR & \% \\
\hline BAJA & 2 & $14.2 \%$ \\
\hline MEDIA & 5 & $35.8 \%$ \\
\hline ALTA & 7 & $50.0 \%$ \\
\hline
\end{tabular}

Fuente: Elaboración propia (2020)

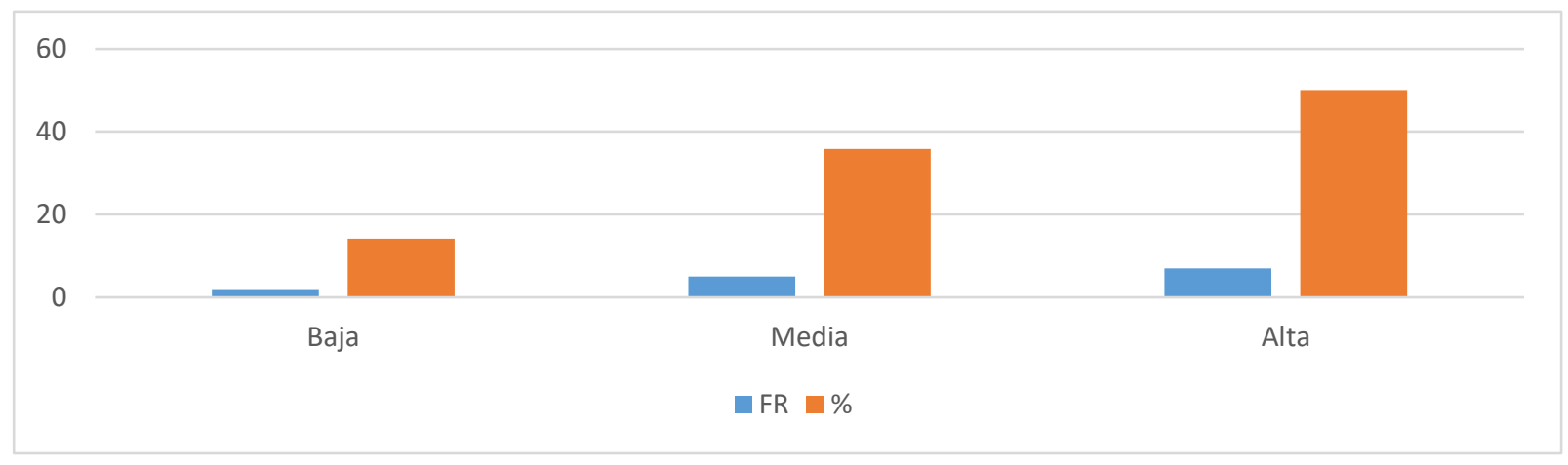


Aida Monserrate Macias-Alvia; Tibisay Rincón-Ríos; Bolívar Cevallos-Jácome; Letty Soraida Quimi-Cobos

\section{Gráfico №6.}

Distribución frecuencial y porcentual según agencia de autocuidado del total de los docentes de la carrera de Enfermería de la Universidad Estatal del Sur de Manabí.

En la tabla y grafico No.6, se presenta la distribución frecuencial y porcentual según agencia de autocuidado del total de los docentes de la carrera de Enfermería de la Universidad Estatal del Sur de Manabí, en la misma se indica que el 50\% de los docentes investigados se ubican en un nivel alto de Capacidad de agencia de autocuidado y el $35.8 \%$ con una capacidad de agencia de autocuidado media y solo el $14.2 \%$ con una capacidad de agencia de autocuidado baja.

\section{CONCLUSIONES}

Los resultados de este estudio indicaron que la capacidad de agencia de autocuidado es deficiente en la mitad de los participantes, al lograr ubicarse entre el nivel medio y bajo de evaluación.

El total de los docentes participan en reuniones o festividades sociales, comparten actividades de recreación con su familia y nunca sustituyen la comunicación directa con su familia por el uso del chat, teléfono o celular.

Nunca en sus mayores porcentajes de opinión por los docentes investigados consumen de forma frecuente medicinas sin recetas médicas, se realizan mamografías y/o papanicolau de forma anual. También, indicaron no sentir que duermen bien y levantarse descansados o conciliar el sueño y realizar siestas diarias.

Siempre la alimentación de los docentes incluyen pescados, raciones de verduras, cereales, menestras y productos lácteos mínimo tres veces por semana y en menor proporción consumen pizzas, hamburguesas, salchipapas y productos que contengan azúcar (golosinas)

\section{FINANCIAMIENTO}


Universidad Nacional Experimental Francisco de Miranda (UNEFM). Santa Ana de Coro. Venezuela

Aida Monserrate Macias-Alvia; Tibisay Rincón-Ríos; Bolívar Cevallos-Jácome; Letty Soraida Quimi-Cobos

No monetaria

\section{AGRADECIMIENTO}

A los docentes de la carrera de Enfermería de la Universidad Estatal del Sur de Manabí; por el apoyo en el desarrollo de la investigación.

\section{REFERENCIAS CONSULTADS}

1. Cacha Antonio A. Papel de enfermería en atención primaria.

2. Sánchez B. Utilidad de los modelos de enfermería en la práctica.[ Utility of nursing models in practice] Bogotá, D.C.: Unibiblos; 2002

3. Bastías, E. y Stiepovich, B. Una revisión de los estilos de vida de estudiantes universitarios Iberoamericanos; Ciencia y enfermería, 20(2): 93-101.2014

4. López Izuel CH. Cuidados enfermeros. Barcelona: Masson; 2005

5. De la Fuente V, Omaña V, Soon Ran R, Alavés C, Peña H y Sierra E. Conocimiento y acciones de los adolescentes sobre el autocuidado de su salud. 2011; Rev. CONAMED, 16(1): 29-33.

6. Naranjo Hernández Y, Concepción Pacheco Ja, Rodríguez Larreynaga M. La Teoría Déficit De Autocuidado: Dorothea Elizabeth Orem. Revista Gaceta Médica Espirituana. 2017; Vol. 19 (3): 1129-1130.

7. Duarte E. y Anderson N. Programa de autocuidado para el mejoramiento de la calidad de vida de atletas universitarios. Revista Enfermería Actual en Costa Rica. 2013; No. 25: 1-13.

8. García, L. Teoría del déficit de autocuidado de Orem. Presentación en diapositivas. Slideshare.

9. Fernández, A. y Manrique, F. Agencia de cuidado. Revista Online: Salud, Historia y Sanidad; 4(1): (enero-abril). Pág. 8. ISSN 1909-2407. Disponible en : https://n9.cl/uf4et 
Universidad Nacional Experimental Francisco de Miranda (UNEFM). Santa Ana de Coro. Venezuela

Aida Monserrate Macias-Alvia; Tibisay Rincón-Ríos; Bolívar Cevallos-Jácome; Letty Soraida Quimi-Cobos

10. Landeros, E. Capacidades de autocuidado y percepción del estado de salud en adultos con y sin obesidad. Tesis para optar al título Magister a Ciencias de Enfermería. Universidad Autónoma de Nuevo León. México. Facultad de enfermería. Disponible en: http://cdigital.dgb.uanl.mx/te/1080124318.pdf.2003

11. Orem, D. Modelos y teorías de enfermería. Barcelona: Harcourt Brace. Orem, D. E. Conceptos de enfermería en la práctica, México: Ediciones Científicas y Técnicas, S.A.1999

12. Suárez R. Autocuidado. Rev. Colombia. Vol.10 .Págs. 4556.

13. Salcedo Álvarez RA, González Caamaño BC, Jiménez Mendoza A, Nava Ríos V, Cortés Olguín DA, Eugenio Retana MB. Autocuidado para el control de la hipertensión arterial en adultos mayores ambulatorios: una aproximación a la taxonomía NANDA-NOC-NIC. 2012; 9(3): 25-43.

14. Díaz Nava J, Márquez Guanipa J. Estimación del potencial de competencias en la implementación de ejes transversales en instituciones de Educación Superior. Sapiens. Revista Universitaria de Investigación, 2007. Año 8 (1): 205-224.

15. Organización Mundial de la Salud (OMS) Determinantes Sociales de la Salud. página institucional electrónica: Disponible en: http://www.who.int/social_determinants/es/ 2016

16. Escobar, M., Franco, Z. y Duque, J. El autocuidado: un compromiso de la formación integral en educación superior. Hacia la Promoción de la Salud, 16(2), 132-146. Disponible http://www.scielo.org.co/scielo.php?script=sci arttext\&pid=S012175772011000200010\&lng=en\&tlng=es. .2011

17. Sanhueza M, Castro M, Merino J. Optimizando la funcionalidad del adulto mayor a través de una estrategia de autocuidado. Av Enferm, 2012; 30 (1). Disponible en: www.index-f.com/rae/301/023031.php.2012

18. Aponte L, Bohórquez L, Fajardo A. Readaptación y validación de la escala "Valoración Agencia de Autocuidado" basada en la teoría de Orem para los trabajadores de la construcción. (tesis). Bogotá: Pontificia Universidad Javeriana; 2011. 
CIENCIAMATRIA

Revista Interdisciplinaria de Humanidades, Educación, Ciencia y Tecnología

Año VI. Vol. VI. N¹. Edición Especial. 2020

Hecho el depósito de ley: pp201602FA4721

ISSN-L: 2542-3029; ISSN: 2610-802X

Universidad Nacional Experimental Francisco de Miranda (UNEFM). Santa Ana de Coro. Venezuela

Aida Monserrate Macias-Alvia; Tibisay Rincón-Ríos; Bolívar Cevallos-Jácome; Letty Soraida Quimi-Cobos

19. Hernández, S. R. Fernández, C. C., Baptista, L.P. Metodología de la Investigación. Quinta edición. Chile: Mc Graw Hill. 2014.

20. Ministerio de Salud Pública. Código de Ética. Ecuador. Disponible en: https://n9.cl/u4xnn

(C2020 por el autor. Este artículo es de acceso abierto y distribuido según los términos y condiciones de la licencia Creative Commons Atribución-NoComercial-Compartirlgual 4.0 Internacional (CC BY-NC-SA 4.0)

(https://creativecommons.org/licenses/by-nc-sa/4.0/). 